\title{
IV Conferencia Internacional de la red de investigadores Trayectorias, 6-10 de octubre de 2021, Heidelberg Akademie der Wissenschaften
}

\author{
por \\ Gracia Llorca Llinares \\ Universidad de Tübingen, Alemania \\ gracia.llorca-llinares@student.uni-tuebingen.de \\ Tim Reichert \\ Universidad de Tübingen, Alemania \\ tim.reichert@student.uni-tuebingen.de
}

La IV Conferencia Internacional de Trayectorias: Translation, Interpretation, Adaptation. Music Between Latin America and Europe 1920 to 2020 tuvo lugar del 6 al 8 de octubre de 2021 en la Heidelberg Akademie der Wissenschaften, Alemania. La jornada se realizó en formato híbrido, lo que permitió la participación online de asistentes de Europa y América Latina. Los miembros de la red Trayectorias moderaron las distintas sesiones de la jornada. La organizadora, Christina Richter-Ibáñez (Tubinga), inauguró la conferencia con un estudio etimológico y semántico de los conceptos translation, interpretation y adaptation usando como ejemplo la Ofrenda Musical BWV 1079 de Johann Sebastian Bach y su difusión mundial en distintos medios y artes.

Isabelle Marc (Madrid), la ponente principal, introdujo el tema de la traducción musical. Marc desarrolló conceptos tomados de sus trabajos acerca de travelling songs, como cultural reception, musical reprise, interlingual cover version y stylistic emulation aplicándolos a "Despacito" de Luis Fonsi y a las distintas versiones de esta canción. Las comunicaciones que le seguían ofrecieron más ejemplos de traducción de música popular y conformaban el primer bloque temático. Julio Ogas (Oviedo) presentó la traducción cultural de la música de Waldo de los Ríos en España. Alicia Pajón (Oviedo) comparó las diferencias entre las canciones en inglés de The Platters y sus versiones en español de Los Cinco Latinos. Jesús Herrera (México) presentó las distintas versiones de la canción "Johnny Get Angry" de Joanie Sommers, centrándose en las traducciones interlingüísticas de esta.

Rosalía Castro Pérez (Oviedo) exploró en su comunicación cuatro versiones diferentes de la canción portuguesa "Construçao" de Chico Buarque y la influencia de las estrategias de traducción en la recepción de esta canción. Kathrin Engelskircher (Maguncia) aplicó los conceptos de traducción intrasemiótica, intersemiótica y transemiótica en The Beatles. En el debate posterior se discutió acerca de las bandas tributo y la inspiración estilística. Abner Pérez (Paderborn) examinó el ensamble "La Fuente" y el bar de salsa "Isla de Cuba" para explicar cómo podían clasificarse en el concepto de comunicación transcultural y qué estrategias de traducción, interpretación y adaptación experimenta la salsa en Alemania. El taller con Sydney Hutchinson (Berlín) adoptó la forma de una lectura parcialmente escenificada en la que intervinieron algunos asistentes de la conferencia y en la que el público pudo experimentar el proceso de traducción del libro Made in Saturn de Rita Indiana.

En el área temática de "canción política”, Stefano Gavagnin (Venecia) presentó distintas versiones y traducciones de "Gracias a la vida" de Violeta Parra y planteó la pregunta acerca de la calidad y la independencia de las versiones y el original. Ádám Ignácz (Budapest) examinó la música popular 
latinoamericana en la Hungría socialista entre los años cincuenta y ochenta. En el debate que cerró el primer bloque temático, los asistentes discutieron en torno al papel de las discográficas en la publicación de versiones traducidas de canciones y los intereses económicos detrás de esto. Se señaló la necesidad de conocer el contexto histórico y político del país en cuestión para entender mejor estas versiones. Es por ello que la diplomacia debe estudiarse en profundidad en relación con los géneros musicales populares.

Ranier Guldin (Lugano) inauguró el segundo bloque temático "Art Music, Translation and Self-Translation" con su ponencia respecto de la práctica de traducción y autotraducción del filósofo y científico de medios de comunicación Vilém Flusser. Mauricio Gómez Gálvez (París) realizó una comunicación acerca del proceso de autotraducción a partir del ejemplo de Alter ego de Patricio Wang. El foco del debate se centró en el cambio de instrumentación y sus consecuencias. Iván César Morales Flores (Oviedo) comparó dos obras de distintos periodos, ambas con el título Bembé: una de Alejandro García Caturla de 1929 y otra de Louis Aguirre de 2008. Ambos, a su manera, eligieron un enfoque contemporáneo occidental para continuar con la traducción musical afrocubana.

Sobre ópera, Omar Corrado (Buenos Aires) presentó la ópera argentina Liederkreis de Gerardo Gandini y examinó las citas musicales de liedery piezas para piano de Schumann en esta obra. Además, tematizó el bilingüismo de la ópera y la relación entre esta y la película Esas cuatro notas de Rafael Filipelli. Quedó claro que cada interpretación o adaptación representa un círculo más amplio en torno al núcleo de Schumann que engloba las interpretaciones anteriores. Marín Liut (Buenos Aires) analizó la red de traducciones de cuatro óperas argentinas: Aliados de Sebastián Rivas, Kamchatka de Daniel D'Adamo, Avenida de los Incas 3518 de Fernando Fiszbein y Cachafaz de Oscar Strasnoy, siguiendo la sociología de la mediación. Liut se ocupó de la distinta recepción de estas óperas entre el público y la crítica de Argentina y de Francia. Judith Romero Porras (París) presentó y analizó las distintas adaptaciones culturales de la ópera Murmullos del Páramo del compositor mexicano Julio Estrada en el contexto alemán, español y japonés.

Daniella Fugellie (Santiago de Chile) analizó varias composiciones seriales latinoamericanas y planteó la pregunta de cómo pueden traducirse las técnicas de composición. Al hacerlo, siempre habría que reflexionar acerca del lenguaje científico y la terminología. Pablo Ernesto Jaureguiberry (Rosario) habló sobre la Trilogía Suiseki de Jorge Horst. Aplicando distintos conceptos como transtextualidad, traducción intrasemiótica e intersemiótica y préstamo, analizó las influencias de la reutilización y adaptación de elementos musicales del Liebeslied de Luigi Nono. En el debate que le siguió los asistentes discutieron en torno a la relación de la música vanguardista con ideas políticas de revolución. En Chile, por ejemplo, a diferencia de Europa, el serialismo se asocia con la izquierda y con el Partido Comunista.

El tercer bloque temático se dedicó a los procesos históricos de los años treinta a los cincuenta. Gabriela Rojas Sierra (La Habana) analizó el papel de los franciscanos en la conservación de la identidad nacional vasco-navarra en La Habana bajo la perspectiva de un proceso de traducción musical. En el debate los asistentes señalaron la necesidad de la recuperación de la historia de la música y la Iglesia en Cuba, que ha sido olvidada u ocultada desde 1959. Bernardino Rodríguez Espejos (México) concluyó presentando al guitarrista clásico Andrés Segovia como traductor de la obra Variations sur "Folía de España" et fugue de Manuel M. Ponce a partir de un análisis de documentos epistológicos y fonográficos. Señaló el papel de las necesidades artísticas del guitarrista en los procesos de composición, interpretación y edición de la obra.

El debate final puso de manifiesto que sigue siendo necesario definir con mayor precisión qué se entiende por "traducción" para poder hablar con mayor precisión del proceso y los tipos de interpretación y adaptación en el ámbito de la musicología. No hay que olvidar que las traducciones y adaptaciones musicales están condicionadas por requisitos prácticos, como las expectativas del público y los instrumentos disponibles. El examen del uso versátil del término "traducción" en diferentes disciplinas académicas abrió nuevas perspectivas acerca de la transferencia de música entre América Latina y Europa en el contexto de esta conferencia multidisciplinar. 